Acta Protozool. (2017) 56: 139-147

\title{
Actinomycin D as an Epimutagen in Protists
}

\author{
Zoya I. USPENSKAYA, Alexander L. YUDIN \\ Institute of Cytology, Russian Academy of Sciences, St. Petersburg, Russia
}

\begin{abstract}
A previously unrecorded property of actinomycin D, a well-known antibiotic, was discovered in the course of long-term genetic research on the amoeba Amoeba proteus and the ciliate Dileptus anser. In these protists actinomycin D can induce an unusual type of hereditary variation, which we refer to as the inheritable destabilization of characters. A number of features indicate that this variation is epigenetic, that is, not caused by mutations in the DNA. Therefore, actinomycin D may be considered as an inducer of epigenetic inheritable changes, in other words, as an epimutagen.
\end{abstract}

Key words: actinomycin D, Amoeba proteus, Dileptus anser, epigenetic variation, epimutagen, inheritable destabilization of characters.

Abbreviations: AmD - actinomycin D, MT - mating type.

\section{INTRODUCTION}

We conducted genetic research on two biologically different and phylogenetically distant protist species, the amoeba Amoeba proteus (Amoebozoa, Tubilinea) and the ciliate Dileptus anser (Rhynchostomatia, Litostomatea) (see Adl et al. 2012). In the course of this research we discovered and studied an unusual type of hereditary variation: inheritable destabilization of several phenotypic characters.

\footnotetext{
Address for correspondence: Zoya I. Uspenskaya, Laboratory of Cytology of Unicellular Organisms, Institute of Cytology, Russian Academy of Sciences, 4 Tikhoretsky Ave., St. Petersburg 194064, Russia; E-mail: zoyaus@mail.ru; Tel.: +7 81229718 36; Fax: +78122973541
}

\section{MATERIALS AND METHODS}

The strains of Amoeba proteus (L, B and C) and Dileptus an$\operatorname{ser}(3,13,153, \mathrm{~B}$ and D) were used. Both amoebas and ciliates were cultured using the conventional technique in Prescott's saline, with Tetrahymena pyriformis $G L$ as a food at $25^{\circ} \mathrm{C}$. The stability of amoebas to the toxic effect of ethanol was assessed by the number of individuals surviving after a 5-minute exposure to $7 \%$ ethanol. In micro-aquaria equal volumes of the culture medium containing amoebae were mixed, and $14 \%$ ethanol solution prepared in the same medium. During the entire exposure period the micro-aquaria were tightly closed with lids. After 15-20 minutes of transfer of the amoebas from ethanol to a fresh culture medium, it was found that the dead amoebae had disintegrated, and the survivors had reached the bottom of micro-aquaria and resumed normal division. Calculation of the number of surviving amoebas was carried out under a binocular magnifier.

In the experiments on the effect of Actinomycin D (AmD, "Serva", Federal Republic of Germany) on samples of strains of amoebas (approximately 50 cells) were placed in a solution of $0,1 \mathrm{mg} / \mathrm{ml}$ of AmD for 6 hours. Amoebas were washed thoroughly of AmD, and then was planted individually into micro-aquaria. Clones were 
cultured from AmD-treated cells by individual cultivation. AmD has an insignificant toxic effect: out of 25 individual cultures 21 clones of strain L were grown, and 17 clones of strain $\mathrm{C}$ were grown.

Dilepti were cultivated according to the same standard procedure. Under such conditions, the ciliates usually divide once per day. Clones used crossing were grown in the laboratory from individuals found at different times in water samples from natural reservoirs, mainly in St. Petersburg and the surrounding Leningrad Region. Crosses of the ciliates were carried out using cells marked with one of the two "parent" clones. For this, tetrahymenas were kept in a suspension of Chinese ink for 1.5-2.0 hours. They were then washed from the medium with mascara and fed to dilepti. The black digestive vacuoles of tetrachimens were distinctly seen in the cytoplasm of the dilepti. Before mixing two complementary clones, one of which was labeled, the cells were transferred to frech medium. The labeled cells were then mixed with unmarked cells of the second "parent" clone (50-100 individuals of each clone) in one container. After 16-18 hours, the formed pairs were removed from the mixture, after their divergence, exconjugant clones were obtained, which were isolated and grown separately. Subsequent testing of young clones for puberty and the type of pairing was performed by crossing the clone with three mature type I, II and III mating clones. Immature clones (true exconjugant clones) were further cultivated as mass cultures, periodically testing them with clones-testers.

The stock solution of AmD at a concentration of $1 \mathrm{mg} / \mathrm{ml}$ was diluted with Prescott's culture medium and put into the cultures of ciliates. Concentrations of 5, 10, 15, 20 and $30 \mu \mathrm{g} / \mathrm{ml}$ were tested. For the main experiments, a concentration $15 \mu \mathrm{g} / \mathrm{ml}$ was chosen. AmD was added into the cultures of the ciliates at the desired final concentration. Microaquaria with dilepti in Prescott's medium were added with $\mathrm{AmD}$ at the above concentration at a ratio of $1: 1$. By adding to the mixture the same AmD solution after one or two days, the antibiotic concentration in the mixture was adjusted almost to that of the working one. For three days the dilepti stayed in the AmD solution without food. Then cells were cleansed of antibiotic with Prescott's medium, placed one at a time, and subclones were cultivated as usual. In total, 20 subclones of each clone were grown in this way. The subclones were tested weekly for mating type with cells of initial clones.

\section{RESULTS AND DISCUSSION}

\section{Inheritable destabilization of characters in amoebae}

Since amoebae are agamic, we used microsurgical transplantation of nuclei for genetic research on these protists. We had at our disposal the collection of laboratory clones and clones isolated from natural populations (strains B, C, L and others). Many of these clones differed by some morphological, physiological or biochemical characters. The differences persisted over many years of culturing and, as shown in nuclear transplantation experiments, were exclusively controlled by the nucleus (Yudin and Sopina 1970; Yudin 1982).
Destabilization of some of these phenotypic characters was first found in clones descended from heterokaryons as an inheritable result of cytoplasm-mediated mutual influence of the nuclei (Kalinina and Yudin 1964; Yudin 1973). The initial material used in these first studies was represented, e.g., by strains $\mathrm{X}$ and $\mathrm{Y}$, which showed an inheritable difference in a marker character. Diheterokaryons $\mathrm{X}_{\mathrm{n}} \mathrm{Y}_{\mathrm{nc}}$ and $\mathrm{Y}_{\mathrm{n}} \mathrm{X}_{\mathrm{nc}}(\mathrm{n}-$ nucleus, $\mathrm{c}-$ cytoplasm) and dihomokaryons $\mathrm{X}_{\mathrm{n}}^{\mathrm{n}} \mathrm{X}_{\mathrm{nc}}$ and $\mathrm{Y}_{\mathrm{n}} \mathrm{Y}_{\mathrm{nc}}$ were obtained. These dikaryons were cut into two uninucleate halves with a fine glass needle on the $5^{\text {th }}$ day after they were obtained (but before their natural division) and a clone was grown from each half. After that, these clones were repeatedly and successively tested for the marker character.

The phenotype of both clones originating from a diheterokaryon changed in almost $100 \%$ of the cases. The changes occurred in both nuclei. They were manifested in the inheritable destabilization of the marker character, which was, as mentioned above, controlled by the nucleus. Successively tested samplings of amoebae from a destabilized clone demonstrated the phenotype of one of the "parent" strains in some cases, the phenotype of the other "parent" strain in some other cases and some intermediate state of the character in some other cases still (though it is uncertain to what extent the behaviour of amoebae in a tested sampling was synchronous). The results of experiments of this kind involving two marker characters, resistance to methionine and resistance to ethanol, are shown in Fig. 1. Clones originating from dihomokaryons remained stable. It was shown that destabilization of other nucleus-controlled characters arose in the same way.

It became clear soon that an inheritable destabilization of characters in amoebae may be caused not only by the mutual influence of the nuclei in heterokaryons but also by diverse external impacts such as X-ray exposure, injections of total RNA obtained from amoebae of the same or a different strain, treatment with actinomycin D or ribonuclease etc. (Kalinina 1967, 1968, 1969; Yudin 1979a, 1982; and others). Taken together, the data on induced destabilization in amoebae suggested that in all the cases the researchers dealt with the same phenomenon.

The data indicated that the arising changes were not associated with mutations since inheritable changes resulting in destabilization of the studied characters in amoebae always arose with a very high frequency (up to $100 \%$ ) under the impact of diverse and often undoubtedly non-mutagenic factors. It was hypothesized that 

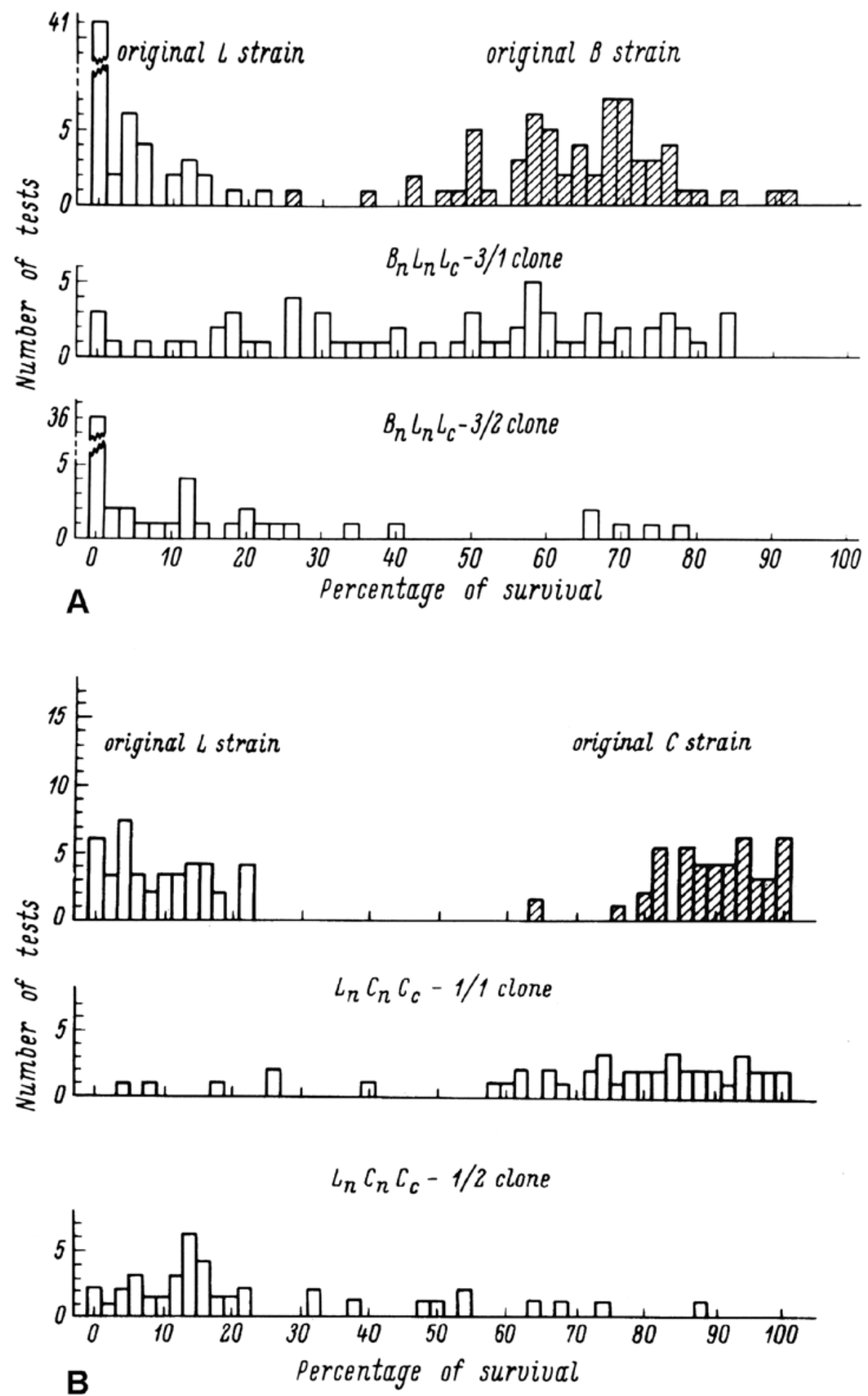

Fig. 1. Examples of inherited instability of traits in amoeba clones grown from mononuclear halves of a diheterokarionic cell (from: Yudin 1967, modified). A - resistance of amoebae to methionine (0.15 M, 18-20 h); B - resistance of amoebae to ethanol (7\%, 5 min). Abscissa: number of amoebae survived of 50 cells taken in each test (in \%); ordinate: number of tests that showed a given survival rate. L, B, and $\mathrm{C}$ - amoebae strains; $\mathrm{n}$, nucleus; c, cytoplasm. 
inheritable characters that can be destabilized are under epigenetic control and that agents inducing inheritable destabilization act upon the mechanisms regulating the activity of genetic material rather than on genetic material itself.

These ideas were based on the concept of epigene (Tchuraev 1975; see also: Monod and Jacob 1961; Jacob and Monod 1963). According to Tchuraev, an epigene is a cyclic system of one, two or several genes that has not less than two stable regimes of functioning retained in the successive series of cell generations. The choice of the functioning regime depends on regulatory molecules of proteins and (or) RNA responsible for regulatory feedbacks in this system. In some cases, the disruption of regulation due to external impacts may result in the switch of the epigenetic system into a new, oscillatory regime, which would also be retained in the series of generations. From this viewpoint, induced destabilization itself has an epigenetic rather than mutational nature. In respect of each character that can be destabilized, the nucleus of the amoeba, while retaining the same genotype, may be in one of the two stable inheritable states or in an inheritable destabilized state (Yudin 1979b, 1982).

The hypothesis of the epigene and the dynamic inheritable memory was developed further in the subsequent years (Golubovsky 1996; Tchuraev 2010). An experimental synthesis of epigenetic systems - importantly, not only toggle switches but also oscillators - considerably reinforced this hypothesis (Judd et al. 2000; Tchuraev et al. 2000, Tchuraev 2010; and others).

\section{Inheritable destabilization of characters in dilepti}

Mating type (MT) is a commonly studied character in sexual ciliates. MTs have been found in dozens of species from all ciliate classes (see for review: Miyake 1996). Since MT systems, their genetic control and the ways in which they are inherited are quite diverse, their study opens promising vistas for comparative genetics. The ciliate Dileptus anser is an especially interesting object because it is a one of the relatively uncommon species excreting specific signal products - mating pheromones triggering the sexual process. The MT of such an organism is determined by the pheromones it excretes and the pheromone receptors it bears on the cell surface (Yudin et al. 1990).

Three MTs have been found in Dileptus anser. Most individuals isolated from natural populations form clones belonging, as revealed by crossing with tester clones, to one of the three MTs. All these MTs are sta- bly inherited in a series of vegetative generations over many years of cultivation under laboratory conditions. Similarly to many other ciliates, dilepti are usually sexually immature after conjugation, that is, unable to conjugate with mature dilepti with complementary MTs (Bleyman 1971, 1972; Nanney 1980; Takagi 1988). After a certain number of cell divisions they become partly mature (adolescent), that is, they can conjugate to only one of the two complementary MTs. Finally, they become fully mature.

The inheritance of MT during crossing in sexual generations was studied with the help of hybridological analysis. The results indicated that this character was inherited synclonally and that it was controlled by a single mat locus with three alleles showing the peck-order dominance: $m a t^{1}>m a t^{2}>m a t^{3}$ (Yudin and Afon'kin 1987).

However, data that did not fit the above scheme were being gradually accumulated. Crossings of various dilepti clones with all the three MTs were performed for several years. On the one hand, some of the crosses continued to demonstrate a synclonal inheritance of this character in sexual generations, peck-order dominance of the alleles and an MT segregation not significantly different from $1: 1$. In other words, we observed, as before, a more or less evident monofactorial Mendelian behaviour of the character whose inheritance was under direct genetic control (Siegel 1970). On the other hand, several crosses showed more or less considerable deviations from this scheme. The anomalous features of these exconjugant clones were as follows: (1) their maturation was delayed as compared to other clones; (2) they temporarily reverted to an immature or adolescent state, that is, a destabilization of the maturity state was observed; (3) some MTs found in them could not be predicted by the scheme described above. Especially interesting observations were made when we repeatedly tested such "anomalous" clones at 1-2 week intervals for a long period of time. MT of many just matured clones appeared to be unstable; when expressed for the first time, it was not uniquely determined and varied with time in one and the same clone. For reasons unknown to us, such change of MT encompassed all cells in a given culture synchronously and unidirectionally. At any rate, in no case was spontaneous selfing (i.e. intraclonal conjugation) observed, which would be expected under asynchronous and/or unlike MT variation within culture. Typically, such an unstable clone, before changing its MT, resumed the state of immaturity or adolescence for a short time when it mated with 
no one tester clone or mated with only one of them, correspondingly.

Examples of the behaviour of these "irregular" clones are given in Table 1. Out of 142 exconjugant clones tested, which were obtained in eight different crosses, a change of MT (that happened once or twice) was noted in 40 clones (28\%). In 30 cases out of 45 we managed to register that a change of MT was accompanied by a transition to the state of immaturity or adolescence. Changes of MT occurred in all the possible directions though with a different probability (Uspenskaya and Yudin 2003; Yudin and Uspenskaya 2006). To illustrate, of 45 MT changes observed 25 were alterations to MT III, but only 8 to MT I. Expression of the most recessive MT seems to be most probable.

It was clear that the causes of unstable MT differentiation in Dileptus anser could have different nature. An epigenetic component of the hereditary MT control could not be ruled out. Based on this, we hypothesised that the mat locus in dilepti has a complex nature, i.e. contains genetic potentiality for all the three MTs (Yudin and Uspenskaya 2007), similarly to the complex MT locus in the ciliate Tetrahymena thermophila (Cervantes et al. 2013). In the course of maturation of an exconjugant dilepti clone, this complex locus undergoes an epigenetic differentiation in the macronucleus (Ma), resulting in the expression of one of the three genetic potentialities encoded in it: an MT-determining "epiallele" of this locus arises. These assumptions help us to explain MT changes in young exconjugant clones as well as deviations from the Mendelian behaviour of the character in crosses. As for the Mendelian behaviour of MT, it appears to be possible only if the complex locus mat can stably differentiate in the generative nucleus, the micronucleus (Mi), resulting in a stable micronuclear epiallele: $m a t^{1}, m a t^{2}$ or $m a t^{3}$.

The origin of a "mutational" allele of the mat locus ensures its stable Mendelian behavior in future crosses. There must be no exconjugant clones unstable for MT in the progeny of the clone in which this kind of differentiation has occurred. But if the micronuclear mat locus is differentiated only functionally, through the repression of any two genetic potentialities out of encoded three (according to the principle of "mutual exclusion" - Nanney 1958), the complexity of the locus at the genetical level is retained; it should be possible to transdifferentiate and change MT into a new, developing Ma. In this case, it must be assumed that differentiation of Mi somehow predetermines the same sense of Ma differentiation. Only with this constraint, oneto-one correspondence between Mi and Ma epialleles would be provided.

\section{Actinomycin D induces inheritable destabilization of characters in amoebae}

As an example, we present here the results of the tests performed on clones grown from AmD-treated amoebae (Yudin 1979a) (Table 2).

Resistance to ethanol (survival after 5 minutes in 7\% ethanol - Yudin and Sopina 1970) was used as a mark-

Table 1. Changes of mating type in just matured exconjugant clones of Dileptus anser (several examples; from: Yudin and Uspenskaya 2007, modified)

\begin{tabular}{|c|c|}
\hline Clone & MT of the clone (Roman numerals) and period of time after conjugation (in brackets, weeks) \\
\hline & Progeny from the cross B (MT I) x D (MT III); a total of 7 clones obtained from this cross were tested \\
\hline $5-14$ & $\mathrm{II}(25-30) \rightarrow \mathrm{IM}(45-50) \rightarrow \mathrm{I}(70-75) \rightarrow \mathrm{I}(95-100)$ \\
\hline $7-3$ & $\mathrm{I}(25-30) \rightarrow \mathrm{IM}(45-50) \rightarrow \mathrm{II}(70-75) \rightarrow \mathrm{II}(95-100)$ \\
\hline $9-7$ & III $(45-55) \rightarrow(70-75) \rightarrow$ II $(95-100)$ \\
\hline $10-21$ & $\mathrm{II}(25-30) \rightarrow \mathrm{IM}(45-50) \rightarrow \mathrm{III}(70-75) \rightarrow \mathrm{III}(95-100)$ \\
\hline \multirow{3}{*}{$13-10$} & Progeny from the cross $\mathrm{F}_{1}$ (MT II) $\mathrm{x}$ B (MT I); a total of 5 clones obtained from this cross were tested \\
\hline & $\mathrm{I}(15-20) \rightarrow \mathrm{IM}(25-30) \rightarrow \mathrm{IM}(45-50) \rightarrow \mathrm{AD}(70-75) \rightarrow \mathrm{II}(95-100)$ \\
\hline & Progeny from the cross $\mathrm{F}_{1}$ (MT II) x D (MT III); a total of 5 clones obtained from this cross were tested \\
\hline $14-2$ & $\mathrm{II}(15-20) \rightarrow \mathrm{IM}(25-30) \rightarrow \mathrm{AD}(45-50) \rightarrow \mathrm{III}(70-75) \rightarrow \mathrm{II}(95-100)$ \\
\hline $18-3$ & $\mathrm{III}(45-50) \rightarrow \mathrm{II}(70-75) \rightarrow \mathrm{I}(95-100)$ \\
\hline $20-2$ & $\mathrm{II}(45-50) \rightarrow \mathrm{AD}(70-75) \rightarrow \mathrm{I}(95-100)$ \\
\hline
\end{tabular}

IM - immaturity (the clone does not mate with any one of three tester clones); $\mathrm{AD}$ - adolescence (clone mates with only one of three tester clones); $\mathrm{F}_{1}$ - progeny from the cross \# B X \# D. 
Table 2. Destabilizing effect of Actinomycin D (1000 $\mu \mathrm{g} / \mathrm{ml}, 2 \mathrm{~h})$ on C amoebae (from: Yudin 1979a, modified)

\begin{tabular}{llll}
\hline Clones & Number of subclones tested & \multicolumn{2}{c}{ Number of subclones with survival rate in 7\% ethanol } \\
\cline { 3 - 4 } & & $<60 \%$ & $60-100 \%$ \\
\hline 1 & 49 & 12 & 37 \\
3 & 43 & 10 & 33 \\
4 & 71 & 6 & 65 \\
5 & 68 & 4 & 64 \\
6 & 24 & 0 & 24 \\
\hline
\end{tabular}

er character. This time, in order to reduce the duration of the experiment, clones were tested not several times successively but just once. Each clone to be tested for stability was used to establish several dozen subclones cultivated in parallel. When the number of cells in each subclone was not less than 50, all these subclones were tested for resistance to ethanol at the same time. If all the subclones showed a resistance typical of the initial strain $\mathrm{C}$, the clone was considered to be stable. If at least some of the subclones showed a resistance not characteristic of strain $\mathrm{C}$, the clone in general was considered to be unstable.

Resistance to ethanol of the initial strain $\mathrm{C}$ in the course of the experiment was as follows: out of the 324 subclones tested, in one clone the survival made up 58\%, while in all the other clones it made up $60-100 \%$, with the survival of $93 \%$ subclones falling within the range of $80-100 \%$. Therefore, survival values of $60-100 \%$ were considered to be typical of strain $\mathrm{C}$.

Four out of the six clones tested showed destabilization (Table 2). The poorest survival values observed in these clones were much smaller than those observed in control amoebae C. Interestingly, we found no indications of a destabilizing effect of the antibiotics emetine $\left(10^{-4} \mathrm{M}, 24 \mathrm{~h}\right)$ and cyclohexamide ("Serva" Federal Republic of Germany, $1000 \mu \mathrm{g} / \mathrm{ml}, 24 \mathrm{~h}$ ) tested in the same experiment.

Destabilizing activity of $\mathrm{AmD}$ in amoebae was also shown in experiments made by our colleagues (Kalinina 1968; Olenov 1970)

\section{Actinomycin D induces inheritable destabilization of mating type in dilepti}

If MT determination in dilepti has an epigenetic nature, mature clones may retain in their MA a genetic potentiality for all the three MT. In such a case they can, in principle, change their MT. This idea was supported by the fact that MT changes in old laboratory clones could be induced by the antibiotic AmD. We would like to emphasize, again, that mature clones of dilepti, both those isolated from natural populations and those cultured in the laboratory, usually retain an unchanged MT over many months and even years of observations. Deviations from this rule are very rare.

Our study showed that the destabilization of mature laboratory clones of dilepti, expressed in MT changes, was indeed possible. We used three laboratory clones of Dileptus anser: no. 3 (MT I), no. 13 (MT II) and no. 153 (MT III). The cells were kept for 3 days in a culture medium with AmD $(15 \mu \mathrm{g} / \mu \mathrm{l})$. Then the cells were rinsed and each single cell was used to establish a subclone. Altogether, 20 subclones of each clone were grown. Subclones of all three clones behaved in a similar manner. Some subclones developed a temporary state of immaturity, which could be more or less prolonged, while some showed a different MT than that of the initial clone; switching to all the MTs possible in dilepti was also recorded in the course of observations. These results are summarized in Table 3.

Thus, there are good reasons to think that AmD destabilizes the MT of mature clones of dilepti. It turns out that the genotype of a long-mature cell, which stably reproduces a given MT, has a hidden genetic potentiality for the other MTs, which may be realized under certain conditions. In a maturing exconjugant clone, a normal epigenetic differentiation of the mat locus in Ma is, for some reasons yet unknown, disrupted and the system switches to an inheritable destabilized regime.

As for the mechanisms of internuclear interactions in ciliates, recently there are data on the information role in them of small RNAs. They relate mainly to internuclear interactions, which control very complex and very precise rearrangements of the germline genome, occurring in the developing Ma after the conju- 
Table 3. Changes in mating type of clone 153 (MT III) Dileptus anser after the action of actinomycin D (15 $\mu \mathrm{g} / \mathrm{ml}, 3 \mathrm{days}$; from: Yudin and Ushpenskaya 2009).

\begin{tabular}{|c|c|c|c|c|c|c|c|c|}
\hline \multirow{2}{*}{$\begin{array}{l}\text { Subclones } \\
\text { of clone } 153\end{array}$} & \multicolumn{8}{|c|}{ Mating type of subclones at consecutive testing (weeks after action) } \\
\hline & $\overline{1-2}$ & $3-4$ & $5-6$ & $7-8$ & $9-10$ & $11-12$ & $13-14$ & More than 15 \\
\hline $153-1$ & III & - & - & $\mathbf{I}$ & I & dead & & \\
\hline $153-2$ & III & - & III & - & II & II & II & II \\
\hline $153-3$ & III & - & - & - & I & I & I & I \\
\hline $153-4$ & - & - & - & III & III & III & III & III \\
\hline $153-5$ & III & III & III & III & III & III & III & III \\
\hline $153-6$ & I & - & - & I & III & III & I & I \\
\hline $153-7$ & III & - & - & - & - & II & - & II \\
\hline $153-8$ & III & III & - & - & - & dead & & \\
\hline $153-9$ & - & - & - & II & II & II & II & II \\
\hline $153-10$ & - & - & - & - & - & dead & & \\
\hline $153-11$ & III & - & - & - & dead & & & \\
\hline $153-12$ & III & - & - & III & III & III & III & III \\
\hline $153-13$ & - & - & I & I & I & I & - & dead \\
\hline $153-14$ & III & - & II & II & - & - & dead & \\
\hline $153-15$ & - & - & III & II & - & - & II & II \\
\hline $153-16$ & III & III & III & III & III & - & - & dead \\
\hline $153-17$ & - & - & II & - & - & I & I & I \\
\hline $153-18$ & III & III & III & III & III & III & III & III \\
\hline $153-19$ & - & - & I & I & - & - & I & I \\
\hline $153-20$ & - & II & - & II & - & II & II & II \\
\hline
\end{tabular}

The dash (- ) indicates that subclone does not react with any of the three test clones (immaturity state). Bold type indicates cases of MT manifestation that are not characteristic of the initial clone 153 .

gation. The most widely discussed is the model of scanning RNAs (Meyer and Chalker 2007; Duharcourt et al. 2009; Nowacki and Landweber 2009).

\section{CONCLUSION}

Studies of non-Mendelian inheritance in protists provided numerous examples of epigenetic heredity based on various epigenetic mechanisms (Nanney 1958, 1980; Beale and Preer 2008). Some of these mechanisms are quite complex (see, e.g.: Kataoka and Mochizuki 2011). In this study, we proceeded from the fact that in two entirely different protist species, an amoeba and a ciliate, epigenetic mechanisms were involved in the control of several inheritable characters (resistance to ethanol and to methionine in amoebae, mating type in dilepti). These mechanisms operated in such a way that the system either remained in one of the two or three alternative stable states ("epialleles") or switched to an oscillatory, unstable regime.

Importantly, it was not only the manifestation of a character over many vegetative generations that was inherited in destabilized clones of amoebae or ciliates. Once destabilization has been induced, it was inherited as well. In other words, an epigenetic system switched from a stable differentiation regime into an inheritable oscillating regime. During long-term observations of destabilized clones, no reversions to stabilization were observed in either amoebae or dilepti.

$\mathrm{AmD}$ is well known in experimental biology as a transcription inhibitor (for the summary of the data on the effect of AmD on A. proteus available by late 1980s see: Yudin 1982, p. 137-138). The inhibition occurs due to the formation of a stable complex between AmD and DNA and the disruption of the DNA-dependent RNA synthesis (Hollstein 1974; Sobell 1985). There are few, if any, data on the mutagenic activity of AmD. If it is a mutagen, it is a very weak one (Fishbein et al. 1970; Fishbein 1979; Koba and Konopa 2005). On the other hand, it is highly probable that $\mathrm{AmD}$, suppressing the synthesis of RNA and, thus, the synthesis of proteins, may disrupt normal regulatory associations in epigenetic contours, bringing about inheritable changes, "epimutations", of epigenetically controlled characters, in particular, their destabilization. In these cases AmD may be considered as an epimutagen, that is, an agent inducing inheritable changes not associated with mutations (Holliday and Ho 2002; Maletskaia et al. 2006; Lamparska et al. 2012; Arai et al. 2015; and others). 


\section{REFERENCES}

Adl S. M., Simpson A. G. B., Lane C. E., Lukeš J., Bass D. et al. (2012) The revised classification of eukaryotes. J. Eukaryot. Microbiol. 59: 429-493

Arai Y., Hayakawa K., Arai D., Ito R., Iwasaki Y. et al. (2015) Putative epimutagens in maternal peripheral and cord blood samples identified using human induced pluripotent stem cells. BioMed. Res. Int. Article ID 876047, doi: 10.1155/2015/876047

Beale G. H., Preer J. R. (2008) Paramecium: Genetics and Epigenetics. CRC Press. Taylor and Francis Group, London, New York

Bleyman L. K. (1971) Temporal patterns in the ciliated protozoa. In: Developmental aspects of the cell cycle, Academic Press, New York, 67-91

Bleyman L. K. (1972) A new spontaneous early mature mutant in Tetrahymena pyriformis. Genetics 71: s5-s6

Cervantes M. D., Hamilton E. P., Xiong J., Lawson M. J., Yuan D., Hadjithomas M., Miao W., Orias E. (2013) Selecting one of several mating types through gene segment joining and deletion in Tetrahymena thermophila. PLoS Biol. 11: e1001518, doi: 10.1371/journal.pbio.1001518

Duharcourt S., de Lepère G., Meyer E. (2009) Developmental genome rearrangements in ciliates: a natural genomic subtraction mediated by non-coding transcripts. Trends Genet. 25 (8): 344-350

Fishbein L. (1979) Potential industrial carcinogens and mutagens. Elsevier Scientific Publishing Company, Amsterdam, Oxford, New York

Fishbein L., Flamm W. G., Falk H. L. (1970) Chemical mutagens. Academie Press, New York, London

Golubovsky M. D. (1996) Epigenes concept 20 years later. Biopolymers Cell 12: 5-24 (in Russia)

Holliday R., Ho T. (2002) DNA methylation and epigenetic inheritance. Methods 27: 179-183

Hollstein U. (1974) Actinomycin. Chemistry and mechanism of action. Chem. Rev. 74 (6): 625-652, doi:10.1021/cr60292a002

Jacob F., Monod J. (1963) Genetic repression, allosteric inhibition, and cellular differentiation. In: Cytodifferentiaton and macromolecular synthesis. New York, London, Academie Press, $30-64$

Judd E. M., Laub M. T., McAdams H. H. (2000) Toggles and oscillators: new genetic circuit designs. BioEssays 22: 507-509

Kalinina L. V. (1967) Hereditary changes caused by x-irradiation in Amoeba proteus. Tsitologiya 9: 1543-1549 (in Russian with English summary)

Kalinina L. V. (1968) Hereditary changes in amoebae caused by actinomycin D treatment. Tsitologiya 10: 1589-1597 (in Russian with English summary)

Kalinina L. V. (1969) A study of the resistance to streptomycin in amoebae. Tsitologiya 11: 760-767 (in Russian with English summary)

Kalinina L. V., Yudin A. L. (1964) Genetic interacnion of nuclei in heterokaryons of amebas. Tsitologiya 6: 695-709 (in Russian with English summary)

Kataoka K., Mochizuki K. (2011) Programmed DNA elimination in Tetrahymena: a small RNA-mediated genome surveillance mechanism. Adv. Exp. Med. Biol. 722: 156-173

Koba M., Konopa J. (2005) Actinomycin D and its mechanisms of action. Postep. Hig. Med. Dosw. 59: 290-298 (in Polish with English summary)

Lamparska K., Clark J., Babilonia G., Bedell V., Yip W., Smith S.S. (2012) 2'-Deoxyriboguanylurea, the primary breakdown product of 5-aza-2'-deoxyribocytidine, is a mutagen, an epimutagen, an inhibitor of DNA methyltransferases and an inducer of 5-azacytidine-type fragile sites. Nucleic Acids Res. 40: 9788 801, doi: 10.1093/nar/gks706

Maletskaia E. I., Iudanova S. S., Maletskiǐ S. I. (2006) Effect of the epimutagen 5-azacytidine on the structure of floral-stalk metameres in sugar beet (Beta vulgaris L) Genetika 42: 939-946 (in Russian with English summary)

Meyer F., Chalker D. L. (2007) Epigenetics of Ciliates. Epigenetics, (Eds. C.D. Allis et al.) CSHL Press, 127-150

Miyake A. (1996) Fertilization and sexuality in Ciliates. In: Ciliates: Cells as organisms (eds. R. Hausmann and P.C. Bradbury), Stuttgard, Jena, New York, Gustav Fischer Verlag, 243-290

Monod J., Jacob F. (1961) General conclusions: teleonomic mechanisms in cellular metabolism, growth, and differentiation. Cold Spring Harbor Symp. Quant. Biol. 26: 389-401

Nanney D. L. (1958) Epigenetic control systems. Proc. Nat. Acad. Sci. U.S.A. 44: 712-716

Nanney D. L. (1980) Experimental Ciliatology. An Introduction to Genetics and Developmental Analysis in Ciliates (eds. John Wiley and Sons), New York, Chichester, Brisbane, Toronto

Nowacki M., Landweber L. (2009) Epigenetic inheritance in ciliates. Curr. Opin. Microbiol. 12: 638-643

Olenov J. M. (1970) Epigenomic variability. Ontogenez 1: 10-16 (in Russian with English summary)

Siegel R. (1970) Genetics of mating types in ciliates. Ontogenez 1: $157-165$

Sobell H. (1985) Actinomycin and DNA transcription. Proc. Nat. Acad. Sci. U.S.A. 82: 5328-5331, doi:10.1073/pnas.82.16.5328

Takagi Y. (1988) Aging. In: Paramecium. Springer-Verlag, Berlin, 131-140

Tchuraev R. N. (1975) The epigene hypothesis. In: Studies on mathematical genetics, Issue of Scientific Papers (ed. Ratner V.A.). Novosibirsk, 77-94 (in Russian with English summary)

Tchuraev R. N. (2010) Epigenes - overgenes level hereditary units. Ecological Genetics 8: 17-24

Tchuraev R. N., Stupak I. V., Tropynina T. S., Srupak E. E. (2000) Epigenes: design and contruction of new hereditary units. FEBS Lett. 486: 200-202

Uspenskaya Z. I., Yudin A. L. (2003) Mating types in the ciliate Dileptus anser. Genetical instability in the mating type system. Tsitologiya 45: 510-514 (in Russian with English summary)

Yudin A. L. (1967) Nuclear-nuclear interactions in amoeba. In: The control of nuclear activity. Englewood Cliffs, New Jersey, Prentice Hall, Inc., 299-318

Yudin A. L. (1973) Nuclear-nuclear interactions in heterokaryons. In: The biology of Amoeba, Academic Press, New York, London, 505-524

Yudin A. L. (1979a) Mechanisms of destabilization of hereditary characters in amoeba. II. Heritable variations induced by some antibiotics. Acta Protozool. 18: 571-580

Yudin A. L. (1979b) Nuclear transplantation studies in Amoeba proteus. Int. Rev. Cytol. Suppl. 9: 63-100

Yudin A. L. (1982) Nucleocytoplasmic relationships and cell heredity in amoebae. Leningrad, Nauka (in Russian)

Yudin A. L., Afon'kin S.Yu. (1987) Genetic determination and inheritance of mating types in Dileptus anser (Gymnostomatida, Holotrricha). In: Current Problems of Protozoology. Nauka, Leningrad, 32 (in Russian)

Yudin A. L., Afon'kin S. Yu., Parfenova E. V. (1990) Mating pheromones in the ciliate Dileptus anser. Tsitologiya 32: 107-116 (in Russian with English summary) 


\section{Actinomycin D as an Epimutagen in Protists 147}

Yudin A. L., Sopina V. A. (1970) On the role of nucleus and cytoplasm in the inheritance of some characters in Amoebae (nuclear transfer experiments). Acta Protozool. 8: 1-39

Yudin A. L., Uspenskaya Z. I. (2006) Mating types in the ciliate Dileptus anser. Inheritance and genetic determination. Tsitologiya 48: 364-374 (in Russian with English summary)

Yudin A. L., Uspenskaya Z. I. (2007) Nuclear differentiation for mating types in the ciliate Dileptus anser. A hypothesis. Cell Biol. Int. 31: 428-432
Yudin A. L., Uspenskaya Z. I. (2009) Change of mating type induced by actinomycin D in the ciliate Dileptus anser. Tsitologiya 51: 84-88 (in Russian with English summary)

Received on $4^{\text {th }}$ March, 2017; revised on $16^{\text {th }}$ October, 2017; accepted on $17^{\text {th }}$ October, 2017 